\title{
A possible strike on postoperative atrial fibrillation with the bullet amiodarone
}

\author{
Spencer J. Melby, MD
}

From the Division of Cardiothoracic Surgery, Washington University in St Louis School of Medicine and Barnes Jewish Hospital, St Louis, Mo.

Disclosures: Author has nothing to disclose with regard to commercial support.

Received for publication Aug 28, 2015; accepted for publication Aug 29, 2015; available ahead of print Oct 26, 2015 .

Address for reprints: Spencer J. Melby, MD, Division of Cardiothoracic Surgery, Washington University, St Louis School of Medicine and Barnes Jewish Hospital, 660 S Euclid Ave, Campus Box 8234, St Louis, MO 63110 (E-mail: melbys@gmail.com).

J Thorac Cardiovasc Surg 2016;151:86-7

0022-5223/\$0.00

Published by Elsevier Inc. on behalf of The American Association for Thoracic Surgery http://dx.doi.org/10.1016/j.jtcvs.2015.08.126

Postoperative atrial fibrillation (POAF) is a common and morbid complication after cardiothoracic surgery that occurs in $20 \%$ to $50 \%$ of patients. ${ }^{1}$ It is associated with increased rates of stroke and death. Despite the widespread use of prophylaxis and treatment strategies, including $\beta$-blockers and amiodarone (both of which have shown promising results in randomized studies), the incidence of POAF has remained constant during the last 20 years. $^{2}$ In this issue of the Journal, Wang and associates present a small randomized trial in which they mixed amiodarone or the steroid triamcinolone acetonide with the surgical adhesive Coseal (Baxter Healthcare Corporation, Deerfield, Ill) and sprayed the preparation on the atria of patients after coronary surgery. They found that POAF was significantly lower in the amiodarone group $(4 / 50$, or $8 \%$ ) than in those who received either triamcinolone $(11 / 50$, or $22 \%)$ or the carrier agent $(13 / 50$, or $26 \%)$. Whereas the amiodarone group enjoyed a reduced rate of POAF, those who received the steroid treatment did not. This finding of the ineffectiveness of the steroid spray may have been due to inadequate dosing or absorption (randomized studies have shown a roughly two-thirds decrease with systemic administration), but it also has other implications: if the inflammation contributing to POAF is a systemic problem, the small local concentration may have been too low to have any effect on a widespread inflammatory response. That finding may provide a clue that POAF comes from a larger systemic generalized inflammatory condition; however, the levels of steroid in the atrial tissue were only slightly higher than they were in the periphery, and definitive conclusions are difficult to make. Furthermore, the general lack of knowledge of the mechanism of POAF limits our ability to understand why any given therapy is or is not effective.

Until we have a better understanding of POAF, we will continue to use the blunt instrument of amiodarone, because it has been shown to be somewhat effective in prophylaxis and treatment. Even with institutional protocols of

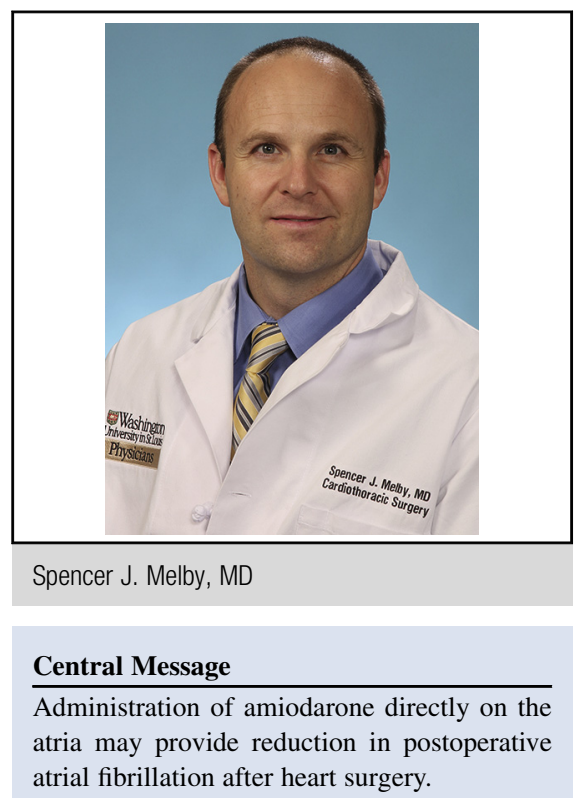

See Article page 80 .

prophylaxis or treatment with amiodarone, POAF remains common among patients after cardiac surgery. Studies that make use of a targeted delivery system such as this one show that high local concentrations of a drug can be achieved while avoiding systemic side effects. This finding has enormous potential for any therapeutic agent because it maximizes treatment while limiting side effects, which in the case of amiodarone it is important, because they are uncommon but serious. Local complications may still occur, however, and in this study bradycardia was more prevalent in the group that received amiodarone. This could prove limiting to widespread use; after the amiodarone preparation has been sprayed on the atrium, it cannot be reversed or removed. Because amiodarone has a half-life of as long as 2 months, this side effect could linger after the patient has been discharged home. This small study cannot tell us whether this is the case; a more adequately powered study is needed.

This small study does demonstrate the ability to achieve direct administration of amiodarone in an easily accessible clinically available adhesive. Achievement of widespread reduction in POAF (such as the two-thirds reduction in this study) would be a major step forward, and larger studies are certainly warranted. Cautious optimism should be maintained, however, as history has shown that few studies with dramatic results actually make a difference in real-world use. 


\section{References}

1. Melby SJ, George JF, Picone DJ, Wallace JP, Davies JE, George DJ, et al. A time-related parametric risk factor analysis for postoperative atrial fibrillation after heart surgery. J Thorac Cardiovasc Surg. 2015;149:886-92.
2. Shen J, Lall S, Zheng V, Buckley P, Damiano RJ Jr, Schuessler RB. The persistent problem of new-onset postoperative atrial fibrillation: a singleinstitution experience over two decades. J Thorac Cardiovasc Surg. 2011; 141:559-70. 